\title{
Preditores de Autocuidado de Mulheres Sadias frente ao Câncer de Colo de Útero
}

\author{
Elisa Kern de Castro \\ Ana Carolina Peuker \\ Fernanda Bittencourt Romeiro \\ Natália Britz de Lima \\ Universidade do Vale do Rio dos Sinos \\ São Leopoldo, RS, Brasil \\ Maria João Figueiras \\ Instituto Piaget \\ Almada, Portugal
}

\begin{abstract}
RESUMO
O autocuidado para a prevenção do câncer de colo de útero está diretamente relacionado ao comportamento ativo da mulher para a realização dos exames preventivos que detectam precocemente a doença. Objetivo: testar um modelo preditivo do autocuidado, considerando percepção da doença e variáveis de comportamento sexual. Participaram 122 mulheres $(M=40,80$ anos; $\mathrm{DP}=12)$, usuárias de serviço público de saúde, do sul do Brasil. Instrumentos: ficha de dados sociodemográficos e clínicos, questionário de percepção da doença e questionário de autocuidado. Resultados: Análise de regressão revelou que o Controle do tratamento e a Idade da primeira relação sexual foram preditores de autocuidado (10,4\% e 4,2\% respectivamente). O modelo explicou $13,1 \%$ da variância do autocuidado. Conclusão: Mulheres que iniciaram a vida sexual mais tarde e com alta percepção de controle do tratamento apresentavam melhor autocuidado. Observa-se que aspectos psicológicos e comportamentais devem ser considerados no delineamento de ações orientadas para o controle da doença.
\end{abstract}

Palavras-chave: Percepção da Doença; Representação da Doença; Câncer; Câncer Cervical; Papiloma Vírus Humano.

\section{ABSTRACT}

\section{Predictors of Self-Care in Healthy Women against Cervical Cancer}

Self-care to prevent cervical cancer is directly related to women's active behaviors to engage in preventive exams to detect the disease early. Purpose: to test a predictive model of self-care, considering the illness perception and sexual behavior variables. Participants: 122 women $(M=40.80$ years old; $S D=12)$ from a public service in southern Brazil. Measures: Sociodemographic and Clinical Data sheet, Illness Perception Questionnaire and Self-care Questionnaire. Results: Linear regression analysis showed that treatment control and age of first sexual intercourse were predictors of self-care (10,4\% e $4,2 \%$ respectively). This model explained $13.1 \%$ of the variance in self-care. Conclusion: Women that had latter sexual initiation and with higher perception that treatment controls illness showed better self-care. It was observed that psychological and behavioral aspects should be considered in design of targeted actions to control cervical cancer.

Keywords: Illness Perception; Illness Representations; Cancer; Cervical Cancer; Human Papilloma Virus.

\section{RESUMEN}

\section{Predictores del Autocuidado de Mujeres Sanas frente al Cáncer Cervical}

El autocuidado para la prevención del cáncer cervial está directamente relacionado a ala conducta activa de las mujeres para la realización de las pruebas preventivas que diagnostican precozmente la enfermedad. Objetivo: Testar un modelo predictivo de autocuidado, teniendo en cuenta la percepción de la enfermedad y variables de la conducta sexual. Participantes: usuarias de servicios de salud público, en el sur de Brasil, 122 mujeres ( $\mathrm{M}=40,80$ años; $\mathrm{SD}=12)$. Instrumentos: Formulario de datos sociodemográficos y clínicos, Cuestionario de la percepción de la enfermedad y Cuestionario de autocuidado. Resultados: El análisis de regresión reveló que el control del tratamiento y la edad en la primera relación sexual fueron predictores de autocuidado (10,4\% y 4,2\% respectivamente). El modelo explicó el $13,1 \%$ de la varianza del autocuidado. Conclusión: Mujeres que comenzaron su vida sexual más tarde y que tenían alta percepción de control del tratamiento presentaron mejor autocuidado. Por lo tanto, los aspectos psicológicos y de comportamiento deben ser considerados en el diseño de acciones dirigidas a controlar la enfermedad.

Palabras clave: Percepción de la Enfermedad; Representación de la Enfermedad; Cáncer, Cáncer Cervical; Virus Del Papiloma Humano. 


\section{INTRODUÇÃO}

O câncer do colo do útero (CCU) possui elevada prevalência no Brasil, sendo foco de políticas nacionais que visam a prevenção da doença, detecção precoce, diagnóstico e tratamento. Mulheres diagnosticadas precocemente e tratadas de forma correta têm praticamente $100 \%$ de chance de cura (INCA, 2014). Embora exista um bom prognóstico do CCU quando diagnosticado nos estágios iniciais, segundo o Instituto Nacional do Câncer (INCA, 2014a), este é o segundo tipo de câncer mais incidente na população feminina e a quarta causa de morte de mulheres por câncer no país.

Inicialmente, é uma doença praticamente assintomática que pode evoluir para sangramento vaginal, secreção vaginal anormal e/ou dor abdominal associada a queixas urinarias ou intestinais (INCA, 2014b). $O$ fator de risco principal é a infecção por subtipos oncogênicos do vírus HPV (Papiloma Vírus Humano) (INCA, 2014c). Além do HPV, existem outros fatores de risco adicionais relacionados com seu aparecimento como: o início precoce da atividade sexual, número elevado de parceiros sexuais, supressão e alteração do estado imunológico, uso prolongado de contraceptivo oral, tabagismo, além de outras influências hormonais (Asiaf, Ahmad, Mohammad, \& Zargar, 2014; INCA, 2014b).

A exposição a certos fatores ambientais e comportamentais durante períodos críticos do desenvolvimento pode repercutir em mudanças epigenéticas persistentes, que podem ter consequências fenotípicas para as células cervicais. Especialistas identificaram que a primeira relação sexual das mulheres jovens é seguida por uma extensa modificação do tecido epitelial cervical, e esse processo pode ser acelerado pelo tabagismo (Hwang et al., 2010; Ma, Collins, Young, Murray, \& Woodman, 2011) e pelo uso de contraceptivo oral. Este processo de maturação associa-se a uma mudança na topografia epitelial cervical, representada pelo aumento da proliferação de células e, portanto, a uma maior vulnerabilidade às lesões ocasionadas pelo HPV (Hwang et al., 2010).

No que concerne à prevenção, o uso de preservativos pode ser uma medida importante para coibir a infecção pelo HPV, mas não totalmente segura. Porém, recentemente, com o desenvolvimento da vacina contra o HPV, prevê-se que o risco de infecção diminuirá no futuro. Segundo o Ministério da Saúde do Brasil, foi disponibilizado em março de 2014 a vacina contra o HPV no SUS (Sistema Único de Saúde), primeiramente nos postos de saúde e escolas públicas e privadas, para meninas de 11 a 13 anos (Brasil, 2014). A vacina contra o vírus HPV é eficaz no que diz respeito à prevenção e proteção de saúde das mulheres que não iniciaram a vida sexual e que, portanto, não tiveram contato com o vírus (Costa \& Goldenberg, 2013).

O exame Papanicolau (exame citopatológico) também é uma forma de prevenção da doença, já que detecta precocemente a alteração celular, e é o exame de rastreamento eleito pelo Ministério da Saúde do Brasil para prevenção do câncer de colo de útero em mulheres que já iniciaram a vida sexual e que tenham entre 25 e 64 anos de idade (INCA, 2014c). No entanto, muitas mulheres brasileiras não realizam o exame Papanicolau. A escassez de recursos financeiros e limitações dos serviços de saúde prejudicam o amplo rastreio do câncer de colo de útero, o que torna seu controle um grande desafio (Albuquerque et al., 2009). A não realização do Papanicolau pode estar relacionada ao medo do exame, dificuldades de acesso aos serviços de saúde (Ferreira \& Oliveira, 2006; Domingos, Murata, Pelloso, Schirmer, \& Carvalho, 2007) e desconhecimento da sua necessidade (Barbeiro, Cortez, Oliveira \& Silva, 2009). Observa-se que quanto mais acesso a informações e melhor status econômico, melhor o autocuidado (Castro \& Moro, 2012).

$\mathrm{O}$ autocuidado se refere a um conjunto de ações realizadas pelo indivíduo para que este consiga manter sua saúde, vida e seu bem-estar. No contexto da saúde e doença crônica, o conceito de autocuidado afastase do modelo médico tradicional de cura. Nesta perspectiva, situa o indivíduo como detentor de um papel ativo de responsabilidade pela sua saúde. Em termos econômicos, o autocuidado desempenha um papel chave, servindo de ponte entre a falta e a demanda pelos serviços de saúde. Ou seja, o indivíduo demanda menos dos serviços de saúde, na medida em que é mais autônomo no seu cuidado em saúde (Castro \& Moro, 2012). Hipotetiza-se que não seja pela falta de conhecimento sobre a importância do Papanicolau, mas a percepção de risco das mulheres e as crenças culturais que afetam os índices de triagem.

Neste contexto, o fato da mulher nunca ou raramente ter feito a triagem para o câncer cervical tem sido relacionado ao conhecimento, atitudes e crenças da mulher sobre sua susceptibilidade e o seu conhecimento sobre a gravidade da doença (Goldman \& Risica, 2004; Waller, McCaffery, \& Wardle, 2004; Sun, Aryee, \& Law, 2007). Entretanto, outro conceito ainda pouco explorado e que pode estar relacionado ao autocuidado é o de percepção da doença. O Modelo de Autorregulação do Comportamento em Saúde ou Modelo do Senso Comum (Leventhal, Nerenz, \& Steele, 1984) postula que as representações ou percepções que o indivíduo tem sobre uma doença, vão influenciar seu comportamento diante dela. Esse modelo se aplica 
tanto para pessoas com alguma doença crônica quanto para pessoas saudáveis (Figueiras \& Alves, 2007).

$\mathrm{O}$ Revised Illness Perception Questionnaire (IPQ-R) tem sido amplamente utilizado para avaliar a percepção da doença em pacientes portadores de doenças crônicas (Jorgensen, Frederiksen, Boesen, Elsass, \& Johansen, 2009; Lee, Cameron, Wünsche, \& Stevens, 2011; Sivell, Marsh, Edwards, Manstead, Clements, \& Elwyn, 2012; Glattacker, Heyduck, \& Meffert, 2012). Este instrumento possui uma versão e adaptação para avaliar a percepção de doença em pessoas saudáveis (Revised Illness Perception Questionnaire for Healthy People; IPQ-RH) realizada por Figueiras e Alves (2007).

A percepção que o indivíduo tem sobre a doença poderá afetar seu comportamento de autocuidado e prevenção frente a ela (Castro \& Moro, 2012). Essas percepções podem ser divididas em cognitivas e emocionais. Adimensão cognitiva inclui a identidade da doença (sintomas e/ou alterações funcionais, associados à determinada doença), percepção das suas causas, duração (cíclica e aguda/crônica), consequências (físicas, psicológicas, sociais, econômicas), e controle (pessoal e do tratamento). Estas percepções sofrem influência do meio no qual o indivíduo está inserido, das experiências pessoais e das informações que este possui sobre a doença (Moss-Morris, 2002; Weinman \& Petrie, 2002; Melo, 2012).

Estudo realizado no Brasil comparou a percepção sobre o câncer de colo de útero em três grupos de mulheres: mulheres com $\mathrm{CCU}$, mulheres com lesões precursoras da doença e mulheres sem lesões (Castro, Aretz, Romeiro, Lawrenz, \& Hass, 2013). Os autores verificaram que, no que concerne à dimensão Identidade, as mulheres sadias percebiam o CCU como mais sintomático e mais cíclico do que as mulheres dos outros dois grupos. Além disso, mulheres com câncer atribuíram mais frequentemente a origem do CCU (causas) a problemas emocionais do que os outros dois grupos estudados. Esses resultados mostram que a experiência de ter CCU possivelmente altera a percepção sobre a doença das mulheres.

$\mathrm{O}$ presente estudo foi desenvolvido com o objetivo testar um modelo preditivo do autocuidado, considerando as dimensões da percepção da doença e variáveis de comportamento sexual (idade da primeira relação sexual e número de parceiros sexuais nos últimos três meses e no último ano). Compreender as percepções de mulheres saudáveis sobre o CCU e como estas representações atuam no comportamento de manutenção da saúde, ou autocuidado, poderá contribuir para o delineamento de estratégias preventivas e orientar a tomada decisões no que concerne às políticas de saúde.

\section{MÉTODO}

\section{Delineamento}

Trata-se de um estudo transversal e preditivo.

\section{Amostra}

Participaram 122 mulheres, com idade média de 40,80 anos $(\mathrm{DP}=12,13)$, usuárias de duas Unidades Básicas de Saúde (UBS) de Porto Alegre. A amostra foi selecionada de forma consecutiva entre as mulheres que compareceram a consultas ginecológicas de rotina nas unidades básicas de saúde, no período de setembro de 2012 a março de 2013 e que tinham entre 18 e 60 anos de idade. A amostra, em sua maioria, foi composta por mulheres que não apresentavam lesões precursoras para o CCU $(73,8 \%)$. A maior parte delas era casada (41\%), com filhos $(78,7 \%)$, trabalhava $(63,9 \%)$ e estudava até o ensino médio (54,1\%).

\section{Instrumentos}

1) Ficha de dados sociodemográficos e clínicos: Este instrumento foi utilizado para descrever as características da amostra, como idade, raça, situação conjugal, escolaridade, situação laboral, renda, além de história clínica e de comportamento sexual. Foram incluídas ainda duas questões sobre a prevenção do câncer. A primeira era Você procura se prevenir contra o câncer de alguma maneira? Com alternativas de resposta: Sim; Se Sim, como se previne?; Não. A outra era uma questão de múltipla escolha denominada Conhecimento sobre o Câncer de Colo de Útero, com as alternativas de resposta: Médico, Família, Escola, Internet, Amigas, Mídias (televisão/jornal/revista) e Outros.

2) Revised Illness Perception Questionnaire for Healthy People (IPQ-RH) (Figueiras \& Alves, 2007). Este instrumento foi originalmente adaptado do IPQ-R (Moss-Morris, 2002) que avalia as crenças sobre determinada doença em indivíduos saudáveis. Inclui as dimensões identidade da doença (ex. $O C C U$ tem como sintoma dores, perda de forças), consequências (ex. O CCU tem sérias consequências econômicas), percurso cíclico da doença (ex. x. Minha doença vai e vem em ciclos), duração agudo/crônica (ex. $O C C U$ dura pouco tempo), controle pessoal ( $O$ doente tem o poder de influenciar a evolução do $C C U$ ), controle do tratamento ( $O$ tratamento do $C C U$ pode controlar a doença), coerência da doença (ex. Os sintomas do $C C U$ me confundem), representação emocional (ex. Fico deprimido quando penso sobre o $C C U$ ) e as causas da doença (ex. O CCU é causado pelo estresse).

As participantes responderam ao instrumento, remetendo-se ao CCU. O IPQ-RH total apresentou um Alpha de Cronbach de 0,76. Os coeficientes das 
dimensões foram respectivamente: 0,33 (duração aguda/crônica); 0,39 (controle pessoal); 0,48 (duração cíclica); 0,61 (controle do tratamento); 0,62 (consequências); 0,77 (coerência da doença); 0,81 (causas da doença); 0,82 (identidade da doença) e 0,90(representação emocional). Foram incluídas nas análises as dimensões que obtiveram Alpha de Cronbach $\geq 0,60$.

3) Appraisal Self Care Agency Scale (ASA-A) (Silva, 2002). Instrumento validado e adaptado para a língua portuguesa como Escala Para Avaliar as Capacidades de Autocuidado (EACAC). Corresponde a 24 itens aos quais se atribuem valor em uma escala Likert de 5 pontos, que varia de Discordo plenamente até Concordo plenamente. A pontuação mínima é de 24 pontos e a máxima de 120 pontos. Quanto mais próximo de 120 pontos, melhores as capacidades de autocuidado. A avaliação do instrumento validado compõe a classificação das capacidades de autocuidado de acordo com a pontuação obtida: 24 a 40 pontos (péssimo), 40 a 56 pontos (ruim), 56 a 72 pontos (regular), 72 a 88 pontos (boa), 88 a 104 pontos (muito boa) e 104 a 120 pontos (ótima). Os itens abordam questões como: disponibilidade, vontade e condições de modificar hábitos de vida, também avalia os cuidados como alimentação, higiene e peso e a busca de uma rede de apoio em caso de dificuldade no processo de autocuidado. $\mathrm{Na}$ análise da confiabilidade o instrumento obteve um coeficiente Alpha de Cronbach de 0,89, o que revela boa consistência interna.

\section{Procedimentos de coleta de dados e éticos}

O projeto foi aprovado pelo Comitê de Ética em Pesquisa da Secretaria de Saúde de Porto Alegre, Processo sob número 001.008580.12.0, registro no CEP número 756, emitido no dia 10/04/12. Antes da aplicação dos instrumentos, as participantes foram informadas sobre os objetivos do estudo, riscos e benefícios em participar, sobre o caráter voluntário da sua participação, garantia de sigilo de seus dados de identificação e assinaram o TCLE. O convite para a pesquisa era realizado após a consulta ginecológica de rotina, e eram encaminhadas para a pesquisa pelo(a) seu/sua médico(a). A aplicação dos instrumentos ocorreu em uma sala reservada e livre de interferências na própria UBS. As entrevistas de coleta de dados duravam, em média, 25 minutos. A coleta de dados foi realizada, através de protocolos padronizados, em contato face a face por entrevistadoras treinadas.

\section{Análise dos dados}

Os dados obtidos foram submetidos à análise estatística descritiva e inferencial. Os dados descritivos foram expressos através de medidas de tendência central (médias) e medidas de dispersão (valor mínimo, máximo, desvio padrão). Empregou-se o Teste de Correlação de Pearson para analisar a força e direção das relações estabelecidas entre as dimensões do IPQRH e as variáveis do comportamento sexual (idade da primeira relação sexual e número de parceiros sexuais nos últimos três meses e no último ano) e entre o IPQ-RH e autocuidado. Utilizou-se a regressão linear (stepwise) para verificar o possível papel preditor das variáveis sociodemográficas, clínicas e das dimensões da percepção da doença para o autocuidado. O Alpha de Cronbach, que mede a consistência interna dos instrumentos bem como das suas dimensões, foi utilizado e considerado aceitável quando o valor era $\geq 0,60$. Com base nesse critério, foram excluídas as dimensões que apresentaram alpha inferior sendo elas: duração aguda/crônica $(0,33)$, controle pessoal $(0,39)$ e duração cíclica $(0,48)$. Todas as análises foram realizadas através do programa estatístico SPSS (Statistical Package for Social Science for Windows) 20.0 , e considerou-se significativo o valor de $\mathrm{p}<0,05$.

\section{RESULTADOS}

\section{Comportamento sexual}

Foram realizadas análises descritivas de variáveis relacionadas à incidência do $\mathrm{CCU}$, a fim de caracterizar a amostra em termos de comportamento sexual e fatores de risco para o CCU. A idade média das participantes era de 40,80 anos $(\mathrm{DP}=12,13)$. A média de idade na menarca foi de 12,56 anos $(\mathrm{DP}=2,00)$, e a média de idade na primeira relação sexual foi de 17,79 anos $(\mathrm{DP}=2,93)$. Nas questões referentes ao comportamento sexual, $69,7 \%(\mathrm{~F}=85)$ das mulheres tiveram relação com apenas um parceiro no último ano. Destas 58,8\% $(\mathrm{F}=50)$ referiram nunca terem usado preservativo nas relações sexuais dos últimos três meses, e $52,9 \%(\mathrm{~F}=45)$ nunca usaram no último ano. $\mathrm{O}$ método de contracepção mais escolhido é o anticoncepcional oral $86,1 \%(F=105)$. Das mulheres que tiveram relação sexual com dois ou mais parceiros $13,3 \%(\mathrm{~F}=17)$ no último ano, $52,9 \%(\mathrm{~F}=9)$ nunca utilizaram preservativo nos últimos três meses e 11,8\% $(\mathrm{F}=2)$ no último ano.

Quando questionadas se realizam prevenção para o câncer em geral, $62,3 \%(\mathrm{~F}=76)$ responderam afirmativamente $(\mathrm{Sim})$. As mulheres referiram se prevenir do CCU através de exames preventivos/ consultas médicas $(43,4 \%)$ e hábitos de vida saudáveis (alimentação exercício físico, proteção contra o sol) $(18,9 \%)$. Nenhuma mulher mencionou a vacina contra o HPV como uma forma de prevenção do CCU. Quanto 
ao conhecimento sobre o $\mathrm{CCU}, 94,3 \%(\mathrm{~F}=115)$ das mulheres afirmaram possuir informação sobre a doença e referiram que este conhecimento é obtido, na maioria das vezes, através do médico $(58,2 \%)$ e da mídia $(48,4 \%)$.

\section{Percepção da doença}

Conforme Tabela 1, são apresentadas as análises descritivas (médias, desvio-padrão, mínimo e máximo) das dimensões da percepção da doença e autocuidado. Considerando a dimensão Identidade, a amostra obteve média de 7,42, indicando que as mulheres atribuíram poucos sintomas ao CCU (dimensão varia de 0 a 17). As demais dimensões foram interpretadas a partir do intervalo de variação de um (Discordo plenamente) a cinco (Concordo plenamente), conforme escala Likert.

TABELA 1

Dados descritivos (médias, desvio padrão, mínimo e máximo) da Percepção da doença (IPQ-RH) e Autocuidado (ASA-A).

\begin{tabular}{lcccc}
\hline & Média & DP & Min. & Max. \\
\hline IPQ-RH identidade & 7,42 & 3,756 & 0 & 17 \\
$\begin{array}{l}\text { IPQ-RH controle do } \\
\text { tratamento }\end{array}$ & 4,17 & 0,515 & 1 & 5 \\
IPQ-RH consequência & 3,86 & 0,745 & 1 & 5 \\
$\begin{array}{l}\text { IPQ-RH coerência da doença } \\
\text { IPQ-RH representação }\end{array}$ & 3,29 & 1,024 & 1 & 5 \\
emocional & 3,17 & 1,177 & 1 & 5 \\
Autocuidado (ASA-A) & 94,45 & 13,873 & 60 & 120 \\
\hline
\end{tabular}

As mulheres consideraram que o tratamento pode controlar a doença, tendo em vista a média superior a quatro. No que se refere às Consequências, a média foi próxima a quatro, ou sejas, as mulheres acreditam que o CCU tem graves consequências. Na dimensão Coerência, as mulheres obtiveram média de 3,29, valor situado acima do ponto médio da escala Likert em que estas mulheres acreditavam que compreendiam a doença. Os dados relativos à dimensão Representação emocional sugerem que as participantes consideram o CCU uma doença ameaçadora considerando a média obtida. Com relação à dimensão Identidade, os sintomas mais frequentes relacionados ao CCU foram: infecções $(86,9 \%)$, perda de peso $(68 \%)$, fadiga/cansaço $(66,4 \%)$ e dor $(66,4 \%)$.

Em relação às respostas espontâneas das participantes sobre as possíveis causas do $\mathrm{CCU}$, a categoria com mais respostas foi Falta de prevenção/ cuidado (não realizar exames periódicos, não ir ao médico, não se cuidar) (30,3\%), seguido de Causas relacionadas à sexualidade/DST's (relação sem preservativo, DST, vida promíscua) (15,6\%). A segunda resposta espontânea mais frequente dada pelas mulheres foi Falta de prevenção/cuidado $(18,9 \%)$ e Não sabe (23\%).

\section{Autocuidado}

Em relação às análises do Appraisal Self Care Agency Scale (ASA-A), a maior parte das mulheres da amostra $(41,2 \%)$ apresentou uma capacidade de autocuidado classificada como muito boa, conforme Tabela 2.

TABELA 2

Classificação das capacidades de autocuidado, conforme pontuação obtida no Appraisal Self CareAgencyScale(ASA-A).

\begin{tabular}{lc}
\hline \multicolumn{1}{c}{ Classificação } & Frequência $n^{\circ}$ \\
\hline Regular & $06(05,0)$ \\
Boa & $35(29,4)$ \\
Muito Boa & $49(41,2)$ \\
Ótima & $29(24,4)$ \\
Total & $122(100,0)$ \\
\hline
\end{tabular}

\section{Análise de correlação e análise preditiva}

Com relação às correlações entre as dimensões do IPQ-RH e o autocuidado (Tabela 3), observou-se que o autocuidado correlacionou-se positivamente e significativamente com a dimensão controle do tratamento $(\mathrm{r}=0,324 ; \mathrm{p}<0,001)$ e com a variável idade da primeira relação sexual $(r=0,227 ; p<0,05)$. As demais correlações são das dimensões do mesmo instrumento (IPQ-RH).

\section{Análise preditiva}

A análise de regressão linear (método por passos) foi realizada a fim de identificar o possível papel preditor das variáveis correlacionadas significativamente com autocuidado (dimensão Controle do Tratamento e Idade da primeira relação sexual) para o Autocuidado. Constatou-se que, no Passo 1 , a dimensão Controle do Tratamento obteve valor $\mathrm{r}^{2}=0,104 ; \beta=0,323$, com nível de significância de $p<0,001$. No Passo 2 foi incluída a variável idade da primeira relação sexual. Neste modelo, a dimensão Controle do Tratamento 
TABELA 3

Correlações de Pearson entre autocuidado (ASA) e as dimensões da percepção da doença (IPQ-RH)

\begin{tabular}{lccccccc}
\hline & Autocuidado & $\begin{array}{c}\text { Controle do } \\
\text { tratamento }\end{array}$ & $\begin{array}{c}\text { Coerência } \\
\text { da doença }\end{array}$ & $\begin{array}{c}\text { Representação } \\
\text { emocional }\end{array}$ & $\begin{array}{c}\text { Idade } \\
1^{a} \text { relação } \\
\text { sexual }\end{array}$ & Consequências \\
\hline Autocuidado & 1 &, $306^{* *}$ &,- 099 &,- 104 &, 142 &, $224^{*}$ &, 047 \\
Controle do tratamento &, $306^{* *}$ & 1 &,- 045 &, 087 &,- 062 &, 074 &, 157 \\
Coerência da doença &,- 099 &,- 045 & 1 &, $342^{* *}$ &,- 029 &,- 096 &, 104 \\
Representação emocional &,- 104 &, 087 &, $342^{* *}$ & 1 &,- 043 &,- 097 &, 246 \\
Consequências &, 047 &, $157^{*}$ &, 104 &, $246^{* *}$ &, 123 &,- 119 & 1 \\
Idade &, 142 &,- 062 &,- 029 &,- 043 & 1 &, $385^{* *}$ &, 123 \\
Idade 1a $1^{\text {relação sexual }}$ &, $224^{*}$ &, 074 &,- 096 &,- 097 &, $385^{* *}$ & 1 &,- 119 \\
Consequências &, 047 &, $157^{*}$ &, 104 &, $246^{* *}$ &, 123 &,- 119 & 1 \\
\hline
\end{tabular}

foi preditora do autocuidado explicando $10,4 \%$ da sua variância $(\beta=0,308 ; \mathrm{p}<0,01)$, e a variável Idade na primeira relação sexual explicou $4,2 \%$ da variância $(\beta=0,205 ; \mathrm{p}<0,005)$. Esse modelo revelou que fortes crenças de que o tratamento pode controlar o CCU e o início mais tardio da vida sexual predizem Autocuidado. O modelo geral testado explicou 13,1\% da variância do Autocuidado.

\section{DISCUSSÃO}

O principal objetivo desse estudo foi avaliar a relação entre a percepção sobre o CCU e o autocuidado em mulheres sadias. Os achados contribuem para a compreensão dos fatores que podem mediar o autocuidado em relação ao CCU. A dimensão Controle do tratamento e a Idade da primeira relação sexual foram preditoras do autocuidado. Estes resultados podem indicar que as mulheres que percebem o tratamento como sendo efetivo no controle da doença e que iniciaram sua vida sexual mais tarde desempenham o autocuidado de forma mais adequada.

Com respeito à variável inserida no modelo de regressão Idade da primeira relação sexual, sabe-se que, do ponto e vista biológico, o início precoce da atividade sexual está relacionado com o CCU (Silva, Guimarães, Barbosa, Pinheiro, \& Maia, 2002; Oliveira et al., 2013; INCA, 2014). Existem evidências de que a partir da primeira relação sexual o tecido cervical sofre alterações, aumentando a vulnerabilidade a lesões por HPV (Hwang et al., 2010). Já em relação aos aspectos de comportamento em saúde, a literatura evidencia essa relação entre a realização do papanicolau e vida sexual ativa, contudo deve-se aprofundar a questão da idade de início da atividade sexual. É preciso compreender em que medida o início precoce da vida sexual repercute no autocuidado.

A análise dos dados relativos ao comportamento sexual da amostra revelou que a maioria das mulheres tinha relações com apenas um parceiro e referiu não utilizar preservativo. Destaca-se a baixa adesão ao uso do preservativo na amostra estudada, tanto nos últimos três meses quanto no último ano. Quanto ao uso do preservativo, há evidência de que a mulher tem dificuldades de impor sua vontade, sofrendo influência do desejo do parceiro em relação ao seu uso (Sousa, Cunha, Ximenes, Pinheiro \& Vieira, 2011). A baixa adesão ao uso do preservativo também pode estar relacionada ao fato da maioria das mulheres da amostra ter parceiro fixo. Desta forma, podem não se perceber em risco de contrair DST's, já que no seu período de latência, o vírus HPV pode ser transmitido sem que a pessoa identifique sinais e sintomas evidentes.

Quando questionadas sobre os possíveis sinais e sintomas do CCU, as mulheres atribuíram a estes: infecções, perda de peso, fadiga/cansaço e dor. Esses achados corroboram a literatura específica da área, que demonstra que as mulheres saudáveis atribuem mais sintomas à doença do que mulheres com CCU e com lesões precursoras (Castro, Aretz, Romeiro, Lawrenz, \& Hass, 2013). Contudo, os sintomas têm início quando a lesão no colo do útero progride, ou seja, é invasiva. Somente no estágio tardio da doença os sintomas aparecem e podem incluir sangramento vaginal anormal, corrimento vaginal persistente e dor durante a relação sexual (American Cancer Society, 2014). Neste sentido, o conhecimento de que o CCU, em sua fase inicial, é uma doença sem sintomas, e que 
o início da lesão cervical tem maior possibilidade de cura sem tratamentos invasivos (ex. cirurgia) deve ser difundido para ampliar a busca pela realização do rastreamento. Por outro lado, os sintomas atribuídos ao CCU podem estar mesclados com os sintomas que as pessoas acreditam que o tratamento para o câncer produz, portanto as crenças sobre os sintomas da doença e do tratamento em oncologia podem ser de difícil compreensão.

A maior parte da amostra referiu saber que a prevenção do CCU é realizada através dos exames de rastreamento, o que condiz com a literatura. Segundo National Cancer Institute, o exame Papanicolau pode reduzir a incidência de câncer como a mortalidade pela doença em 80\% (National Cancer Institute, 2014). No Brasil, este exame é a principal forma de rastreamento da doença (INCA, 2014c). Observou-se ainda uma alta taxa de adesão ao exame na população estudada. Entretanto, este dado pode estar superestimado, pois a coleta de dados foi realizada na unidade básica de saúde na qual essas mulheres estavam realizando atendimentos ginecológicos de rotina. Por se tratar de uma amostra clínica, é provável que estes números sejam superiores àqueles encontrados na população geral (Albuquerque et al., 2009).

$O$ fato de nenhuma mulher ter citado a vacina contra o HPV como forma de prevenção do CCU pode sugerir desconhecimento sobre esta nova estratégia preventiva. Medidas educativas devem estar associadas às campanhas de divulgação da vacina e incorporadas na prática clínica dos profissionais de saúde. O amplo conhecimento sobre a vacina se faz necessário, mesmo entre mulheres com idade acima da preconizada para vacinação (de 09 a onze anos). Pois, estas mulheres podem servir de multiplicadoras desta informação em suas comunidades e família (ex. informando filhas, primas, noras, netas, etc) e, assim, favorecer a adesão às campanhas de vacinação $\mathrm{e} o$ controle efetivo do CCU.

Com relação às causas da doença, o CCU é multicausal, mas a infecção pelo HPV é a principal causa, estando presente em 90\% dos casos (INCA, 2014a). A maior parte da amostra, não relacionou o HPV ao CCU. Para elas, a doença estava relacionada à falta de prevenção/cuidado e a outras causas relativas à sexualidade/DST's. Esse dado pode sugerir um desconhecimento sobre a íntima relação do HPV com o CCU. Isso converge com a literatura específica da área que aponta que as mulheres, muitas vezes, desconhecem tal relação. Além disso, algumas mulheres podem não saber sobre o modo de transmissão do HPV e a sua possível ação no organismo (França, França, \& Moraes 2013).
A crença no Controle do Tratamento como preditor de autocuidado frente ao CCU é um dado novo, uma vez que mostra que, mesmo acreditando no tratamento, as mulheres não querem passar por ele. Apesar da efetividade do tratamento e do aumento da sobrevivência dos pacientes com câncer, ele está associado geralmente a muita dor física, psicológica e efeitos colaterais. Figueiras e Alves (2007), demostraram que pessoas saudáveis que acreditam que o tratamento é efetivo no controle da doença, percebem menos consequências, menor duração crônica e menor número de sintomas. É possível que esta percepção positiva sobre a doença possa favorecer que estas pessoas engajem-se em estratégias de autocuidado de forma assertiva. Nesse sentido, a crença de que o tratamento controla o CCU poderia também estar associada, possivelmente, à crença de que o autocuidado previne o CCU. Dado semelhante já havia sido encontrado por Buick e Petrie (2002) com mulheres saudáveis sobre o câncer de mama. Esses autores verificaram que as mulheres que tinham alta percepção de controle do tratamento aderiram melhor ao autoexame das mamas do que aquelas com baixa percepção de controle.

Os achados obtidos neste estudo devem ser interpretados de forma parcimoniosa em virtude da não aleatoriedade da amostra. Por isso, futuras pesquisas devem contemplar um método de amostragem mais robusto, como a seleção randômica de participantes. Além disso, deve-se observar que as mulheres foram convidadas a participar do estudo enquanto aguardavam a consulta ginecológica. Portanto, trata-se de uma amostra que, em algum grau, revelou preocupação com sua saúde buscando auxílio médico. Este fato pode diferenciar essa amostra daquela encontrada na população geral, que nunca buscou atendimento médico especializado. Nesse sentido, novos estudos poderão explorar possíveis diferenças no que concerne ao comportamento de saúde entre mulheres que buscam atendimento ginecológico e aquelas que não o fazem. Dessa maneira, amplia-se a possibilidade da caracterização da percepção da doença, condutas sexuais e de autocuidado de mulheres frente ao CCU.

\section{CONSIDERAÇÕES FINAIS}

O presente estudo põe em evidência que fatores subjetivos - a crença no controle do tratamento - e comportamentais - início da vida sexual mais tardio têm influência na forma como as mulheres se cuidam e se previnem do câncer de colo de útero. Esse resultado é importante porque mostra que a crença no tratamento da doença não impede a sua prevenção, ao 
contrário, a crença no tratamento prediz um melhor cuidado com a saúde. Contudo, é importante destacar que, em alguma medida, as mulheres não devem atribuir todo o controle da doença ao tratamento, pois se eximiriam de condutas de autocuidado dependentes delas (ex. higiene íntima adequada, número de parceiros sexuais). Além disso, os resultados apontam para a necessidade campanhas de conscientização das mulheres em relação à uma vida sexual responsável, o que inclui a conscientização sobre as formas de contaminação pelo HPV, os fatores de risco associados e formas de prevenção do CCU.

É essencial que as mulheres conheçam não só a relação entre CCU e HPV, mas também saibam que existem fatores de risco comportamentais associados ao CCU que elas podem manejar. Estes dados apontam que aspectos psicológicos e fatores comportamentais também devem ser considerados no delineamento de ações orientadas à políticas públicas de saúde tanto para a prevenção, como para o tratamento da doença.

\section{REFERÊNCIAS}

Albuquerque, K. M., Frias P. G., Andrade C. L. T., Aquino E. M., Menezes G., \& Szwarcwald C. L. (2009). Cobertura do teste de Papanicolau e fatores associados à não-realização: um olhar sobre o Programa de Prevenção do Câncer do Colo do Útero em Pernambuco, Brasil. Cad. Saúde Pública, 25(2), 301-9. http://dx.doi.org/10.1590/S0102$311 \mathrm{X} 2009001400012$

American Cancer Society (2014). Signs and symptoms of cervical cancer. Acessado em: 14 mar. 2014, disponível em: http://www.cancer.org/cancer/cervicalcancer/moreinformation/cervicalcancerpreventionandearlydetection/cervicalcancer-prevention-and-early-detection-cervical-cancer-signs-and-symptoms

Asiaf, A., Ahmad S. T., Mohammad S. O., \& Zargar M. A. (2014). Review of the current knowledge on the epidemiology, pathogenesis, and prevention of human papillomavirus infection. Eur J Cancer Prev, 23(3), 206-24. http://dx.doi. org/10.1097/CEJ.0b013e328364f273

Barbeiro, F., Cortez E., Oliveira P., Silva A. (2009). Conhecimentos e práticas das mulheres acerca do exame papanicolau e prevenção do câncer cérvico-uterino. Rev. De Pesq: Cuidado é Fundamental, 1(2), 414-22. Acessado em: 04 abr. 2014, disponível em: http://www.seer.unirio.br/index.php/cuidadofundamental/article/view/419/401

Brasil. Portal Brasil. Ministério da Saúde lança campanha de vacinação contra HPV. Acessado em: 31 mar. 2014, disponível em: http://www.brasil.gov.br/saude/2014/01/ministro-da-saude-faz-pronunciamento-sobre-vacinacao-contra-hpv

Buick, D. L. \& Petrie K. J. (2002). "I know just how you feel”: The validity of healthy women's perceptions of breast-cancer patients receiving treatment. Journal of Applied Social Psychology, 32(1), 110-23. http://dx.doi. org/10.1111/j.1559-1816.2002.tb01422.x

Castro, E. K. \& Moro L. (2012). Factores psicosociales relacionados con el autocuidado en la prevención, tratamiento y postratamiento del câncer de mama. Psicooncología, 9(3), 453-65.

Castro, E. K., Aretz, M., Romeiro F. B., Lawrenz P., \& Hass S. A. (2013). Illness perceptions in brazilian women with cervical cancer, women with precursory lesions and healthy women. Psicooncología, 10(2-3), 417-23. http://dx.doi. org/10.5209/rev_PSIC.2013.v10.n2-3.43459

Costa, L. A. \& Goldenberg P. (2013). Papilomavírus humano (HPV) entre jovens: um sinal de alerta. Saúde e Sociedade, 22(1), 249-26. http://dx.doi.org/10.1590/S0104-12902013000100022

Domingos, A. C. P., Murata I. M. H., Pelloso S. P., Schirmer J., \& Carvalho M. D. (2007). Câncer do Colo do Útero: Comportamento preventivo de auto-cuidado à saúde. Cienc. Cuid. Saúde, 6(2), 397-403. http://dx.doi.org/10.4025/ cienccuidsaude.v6i0.5337

Ferreira, M. L. \& Oliveira C. (2006). Conhecimento e significado para funcionárias de indústrias têxteis sobre prevenção do câncer do colo-uterino e detecção precoce do câncer de mama. Revista Brasileira de Cancerologia, 52(1), 5-15.

Figueiras, M. J. \& Alves N. C. (2007). Lay perceptions of serious illnesses: An adapted version of the Revised Illness Perception Questionnaire (IPQ-R) for healthy people. Psychologyand Health, 22(2), 143-58. http://dx.doi. org/10.1080/14768320600774462

França, M. C. A., França M. C. S., \& Moraes S. D. (2013). Conhecimento de mulheres acerca do papilomavírus humano e sua relação com o câncer de colo uterino. CogitareEnferm, 18(3), 509-14. http://dx.doi.org/10.5380/ce.v18i3.33564

Glattacker, M., Heyduck, K., \& Meffert, C. (2012). Illness beliefs, treatment beliefs and information needs as starting points for patient information - Evaluation of an intervention for patients with chronic back pain. Patient Education \& Counseling, 86(3), 378-389. http://dx.doi.org/10.1016/j.pec.2011.05.028

Goldman, R. \& Risica P. (2004). Perceptions of breast and cervical cancer risk and screening among Dominicans and Puerto Ricans in Rhode Island. Ethnicity and Disease, 14(1), 32-42.

Heino R. K., Kosunen E., \& Rimpela M. (2003). Pubertal timing, sexual behaviour and self-reported depression in middle adolescence. Journal of adolescence, 26(5), 531-45. http://dx.doi.org/10.1016/S0140-1971(03)00053-8

Hwang, L. Y., Ma Y., Benningfield S. M., Clayton L., Hanson E. N., Jay J., Jonte, J., Godwin, M. C., \& Moscicki, A. B. (2010). Factors that influence the rate of epithelial maturation in the cervix in healthy young women. Journal of Adolescent Health, 44(2), 103-10. http://dx.doi.org/10.1016/j.jadohealth.2008.10.006 
Instituto Nacional do Câncer (2014a). Programa Nacional de Controle do Câncer do Colo do Útero - Fatores de Risco. Acessado em: 11 mar. 2014, disponível em: http://www2.inca.gov.br/wps/wcm/connect/acoes_programas/site/home/ nobrasil/programa_nacional_controle_cancer_colo_utero/fatores_risco

Instituto Nacional do Câncer (2014b). INCA e Ministério da Saúde apresentam estimativas de câncer para 2014. Acessado em: 13 mar. 2014, disponível em: http:/www2.inca.gov.br/wps/wcm/connect/agencianoticias/site+/home+/ noticias/2013/inca_ministerio_saude_apresentam_estimativas_cancer_2014

Instituto Nacional do Câncer (2014c). Programa Nacional de Controle do Câncer do Colo do Útero - Detecção Precoce. Acessado em: 20 mar. 2014, disponível em: http://www2.inca.gov.br/wps/wcm/connect/acoes_programas/site/home/ nobrasil/programa_nacional_controle_cancer_colo_utero/fatores_risco

Jorgensen, I., Frederiksen, K., Boesen, E., Elsass, P., \& Johansen, C. (2009). An exploratory study of associations between illness perceptions and adjustment and changes after psychosocial rehabilitation in survivors of breast cancer. ActaOncologica, 48(8), 1119-1127. http://dx.doi.org/10.3109/02841860903033922

Lee, T. J., Cameron, L. D., Wünsche, B., \& Stevens, C. (2011). A randomized trial of computer-based communications using imagery and text information to alter representations of heart disease risk and motivate protective behaviour British Journal Of Health Psychology, 16(1), 72-91. http://dx.doi.org/10.1348/135910710X511709

Leventhal, H., Nerenz D. L., \& Steele D. J. (1984). Illness representation and coping with health threats. In A. Baum, S. Taylor, J.E. Singer, (Eds.). Handbook of Psychology and Health (pp. 219-52). Nova York: Erlbaum.

Ma, Y. T., Collins S. I., Young L. S., Murray P. G., \& Woodman C. B. (2011). Smoking initiations is followed by the early acquisition of epigenetic change in cervical epithelium: a longitudinal study. British Journal of Cancer, 104(9), 1500-04. http://dx.doi.org/10.1038/bjc.2011.113

McKenzie, C. (2009). Exploring perceptions of coronary heart disease in africanamerican women with type 2 diabetes [dissertação]. Chapel Hill: University of North Carolina. http://dx.doi.org/10.1177/0145721710374652

Melo, S. C. (2012). Relação entre percepção da tensão arterial elevada e o estilo de vida adotado: estudo exploratório [dissertação]. Porto: Universidade Fernando Pessoa.

Moss-Morris, R., Weinman J., Petrie K. J., Horne R., Cameron L. D., \& Buick, D. (2002). The Revised Illness Perception Questionnaire (IPQ-R). Psychology and Health, 17(1), 1-16. http://dx.doi.org/10.1080/08870440290001494

National Cancer Institute (2014). Cervical Cancer Prevention. http://www.cancer.gov/cancertopics/pdq/prevention/ cervical/HealthProfessional Acessado em 6/04/2014

Oliveira G. R., Vieira V. C., Barral M. F., Döwich V., Soares M. A., Conçalves C. V., \& Martinez, A. M. B. (2013). Fatores de risco e prevalência da infecção pelo HPV em pacientes de Unidades Básicas de Saúde e de um Hospital Universitário do Sul do Brasil. Rev Bras Ginecol Obstet, 35(5), 226-32. http://dx.doi.org/10.1590/S0100-72032013000500007

Silva T. T., Guimarães M. L., Barbosa M. I., Pinheiro M. F., \& Maia A. F. (2002). Identificação de tipos de papilomavirus e de outros fatores de risco para neoplasia intra-epitelial cervical. Rev Bras Ginecol Obstet, 28(5), 285-91. http:// dx.doi.org/10.1590/S0100-72032006000500004

Silva, J. V. (2002). Adaptação cultural e validação do instrumento de capacidade de autocuidado do "Appraisalof selfcareagencyscale” [dissertação]. São Paulo: Universidade de São Paulo, Escola de Enfermagem.

Sivell, S., Marsh, W., Edwards, A., Manstead, A. R., Clements, A., \& Elwyn, G. (2012). Theory-based design and field-testing of an intervention to support women choosing surgery for breast cancer: BresDex. Patient Education \& Counseling, 86(2), 179-188. http://dx.doi.org/10.1016/j.pec.2011.04.014

Sousa, L. B., Cunha D. F., Ximenes L. B., Pinheiro A. K., \& Vieira N. F. (2011). Conhecimento, atitudes e práticas de mulheres acerca do uso do preservativo. Rev. Enferm. UERJ, 19(1), 147-52.

Sun, L. Y., Aryee S., \& Law K. S. (2007). High-performance human resources practices, citizenship behaviour, and organisational performance: A relational perspective. Academy of Management Journal, 50(3), 558-77. http://dx.doi org/10.5465/AMJ.2007.25525821

Waller, J., McCaffery K., \& Wardle J. (2004). Beliefs about the risk factors for cervical cancer in a British population sample. Preventive Medicine, 38(6),745-53. http://dx.doi.org/10.1016/j.ypmed.2004.01.003

Weinman J., \& Petrie K. J. (2002). Illness perceptions: A new paradigm for psychosomatics? In D. Marks (Ed.). The health psychology reader (pp. 250-4). Londres: SAGE. http://dx.doi.org/10.1016/S0022-3999(96)00294-2

\footnotetext{
Autores:

Endereço para correspondência:

Elisa Kern Kern de Castro

Unisinos, 950 - Cristo Rei

CEP 93022-000 São Leopoldo, RS, Brasil
}

ELISA KERN DE CASTRO - Doutora pela Universidade Autônoma de Madri, Professora da Universidade do Vale do Rio dos Sinos.

Ana Carolina Peuker - Doutora pela Universidade Federal do Rio Grande do Sul, Bolsista Docfix da Universidade do Vale do Rio dos Sinos. FERNANDA ROMEIRO - Iniciação cientifica, Universidade do Vale do Rio dos Sinos.

NATÁLIA BRITZ DE LIMA - Iniciação cientifica, Universidade do Vale do Rio dos Sinos.

MARIA JoÃo FIGUEIRAS - Doutora pela Universidade de Londres, Professora do Instituto Piaget (Portugal)

Recebido em: 21.08 .2014

Aceito em: 05.05.2015

Psico, Porto Alegre, v. 46, n. 3, pp. 331-339, jul.-set. 2015 